\title{
The Analysis of Weak Magnetic Inspection of Standard Test Block Defects
}

\author{
Yuelin Tang1, a , Dingdong Zou, Kunyu Zhang, Chaojun Luo, Minxu Deng \\ ${ }^{1}$ Chongqing Special Equipment Inspection and Research Institute, Chongqing 401121, China \\ atonnyue@163.com
}

\begin{abstract}
Keywords: nondestructive testing; weak magnetic inspection; defections
Abstract. The evaluation of standard test blocks plays an important role in nondestructive test, quality of which influences the inspection and production activities. The weak magnetic inspection is adopted, which direct at the common defections including wracks and holes to achieve the quantitative analysis of standard test block, regarding the split test plate as the detected objects. The standard test block is scanned horizontally and respectively by single detector, dual detector and triple detector. The collected data is analyzed and the experimental results are compared. The result shows that the method not only can probe out the location of defects, but also can take the quantitative analysis of those defections within a given range of error.
\end{abstract}

\section{Introduction}

Special equipment has the characteristics of life safety and high risk, which safety inspection is very important, especially non-destructive testing. As a new non-destructive testing method, the weak magnetic detection technology has a wide range of applications because of its simple operation steps, convenient and easy to learn, no need for coupling agents, and two-dimensional imaging. How to apply weak magnetic detection to special equipment is also a research hotspot ${ }^{[1-2]}$. Among them, standard test blocks are often used for evaluation and calibration. The standard test block means that its material, shape, geometric size, performance are regulated by the authority. It is mainly used to determine the detection sensitivity, the performance of the instrument and probe, adjust the scanning speed and judge the defect size ${ }^{[3]}$. On the one hand, the production of standard test blocks is complicated, as some defects such as shape and size may occur due to fatigue and corrosion. As these defects are often difficult to be perceived by the naked eye, the detection accuracy maybe reduce. On the other hand, due to the high requirements of the standard test block on the size, artificial defects and other structures, the standard test block production cost is high. Therefore, in terms of economics and quality and safety, it is very important to ensure the integrity of standard blocks and to detect various defects in time.

At present, there are detection methods such as penetration detection ${ }^{[4]}$, magnetic particle detection $^{[5]}$, eddy current detection and ultrasonic detection ${ }^{[6]}$. Although the defect area can be estimated, there are some limitations, such as complex operation, high requirements for inspectors. So the further research is needed to accurately achieve quantitative analysis of defects. Compared with other detection methods, weak magnetic detection has the characteristics of simple operation, low energy consumption, intuitive and effective.

In this paper, based on the principle of weak magnetic detection, the split test plate $\mathrm{D}$ is taken as the detection object, and the application of the weak magnetic detection technology in the standard test block is emphasized. By comparing the test results with the actual hole length and width data, the feasibility of non-destructive testing of standard test blocks is explored, and the effectiveness of the method is verified and its influencing factors are analyzed. 


\section{System Measurement Principle}

Weak magnetic detection means that when the magnetic field strength is less than $H_{a m}$, the test piece detection belongs to the field weakening detection range. Weak magnetic detection does not require a magnetizing device to magnetize the test piece to a saturated state. During the detection process, the magnet with small magnetic force runs together with the sensor, and the magnetization and detection are integrated ${ }^{[7-8]}$. Weak magnetic detection is a feature that reflects defects in a stable environment such as the earth's magnetic field by changing the magnetic permeability due to defects. The relative magnetic permeability of the geomagnetic field can be regarded as $\mu^{\prime}$. When the object with relative magnetic permeability $\mu \mu$ is placed smoothly in the earth's magnetic field, the magnetic field lines pass through the workpiece to be inspected. If the material inside is intact, the material is even and continuous. The magnetic lines of force of the workpiece are uniform and the direction is parallel to the surface of the object. However, when a defect occurs inside the test object, the defect causes a change in magnetic permeability ${ }^{[9]}$. When $\mu^{\prime}>\mu$, the magnetic induction line has no effect on the defect, and the magnetic induction line at both ends of the defect at the edge of the workpiece is denser, and the magnetic induction line is convex upward when an abnormality occurs. When $\mu<\mu$, the magnetic induction line has an attractive effect on the defect. In this experiment, when the defect exists, the magnetic induction line is sunken downward. The experiment uses the magnetic induction sensor to test the change of the magnetic induction intensity to reflect the abnormal protrusion and depression of the magnetic induction line to find the defect ${ }^{[10]}$. Under different conditions of defect relative permeability $\mu^{\prime}$, the magnetic field line distribution is shown in fig. 1 .

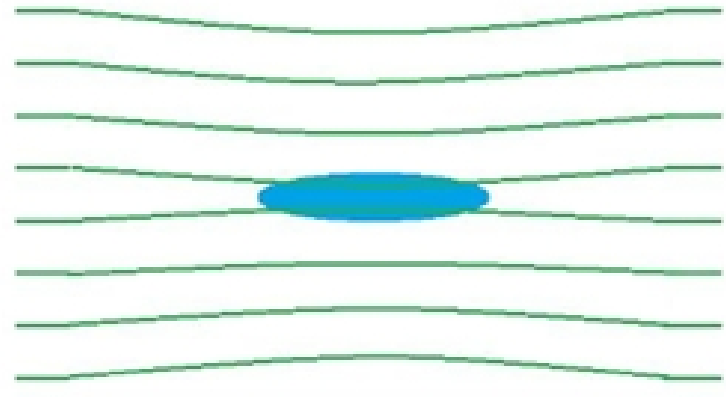

a. $\mu^{I}>\mu$

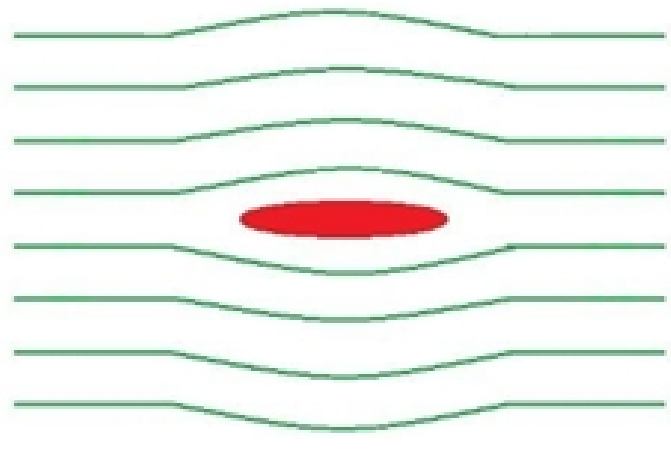

b. $\mu^{t}<\mu$

Fig. 1. Schematic diagram of magnetic field line distribution

The intensity decay of the magnetic field is given below ${ }^{[11]}$.

$$
H=H_{0} \times e^{-\chi T} .
$$

As

$B=\mu_{r} H$.

So

$$
B=B_{0} \times e^{-\chi T} .
$$

where, $H$ is the magnetic field strength after passing the sample, $H_{0}$ is the original magnetic field strength, $B$ is the magnetic induction after the sample is tested, $B_{0}$ is the magnetic induction after the sample is tested, $\mu_{r}$ is the relative permeability, $T$ is the thickness of the sample to be tested.

The susceptibility parameter can be calculated from a sample based on the intensity of the change in the earth's magnetic field, and the resulting image can be used to observe changes in the magnetic 
susceptibility parameter. During the detection process, a sensor is used to detect several points, and the corresponding value of each detection point has a significant magnetic susceptibility. Therefore, the data obtained after the detection can be represented by a matrix ${ }^{[12]}$.

\section{Test and Results}

The test object used in this experiment is the split test plate $\mathrm{D}(400 \mathrm{~mm} \times 400 \mathrm{~mm})$, which have 4 holes,

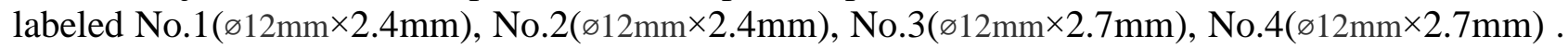
The experimental system consists of an array magnetic probe, a data acquisition device and a computer. The probe receives the magnetic signal and converts it into an electrical signal. The micro-magnetic detector receives the analog signal and converts it into a digital signal, which is processed by the host computer and displayed.

The split test block is placed on the test bench. When the signal is collected, the probe is perpendicular to the test block surface and the probe center is aligned with the defect center line. The array type magnetic probe is manually pushed to move from left to right, and the linear motion is maintained as much as possible during the sampling process. The experiment found that the standard test block used has other unknown defects will affect the judgment of the hole defect signal, so this experiment only uses one artificial hole at a time. To enhance the accuracy of the data, each set of data was collected three times. The signal diagram obtained by scanning the test block separately for the triple probe is show in Fig. 2.
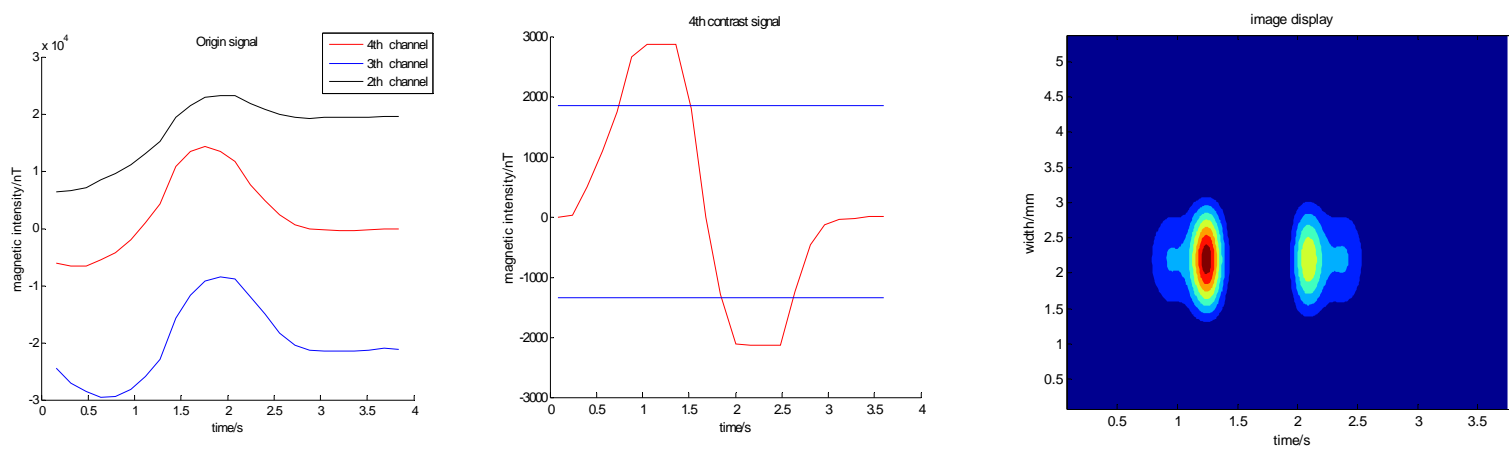

Fig. 2. Results of triple probe detection

The length of the channel signal is calculated through exceeding the threshold portion and multiply the scan speed to obtain the defect length. The inversion method is used to quantitatively analyze the depth of the buried depth. The final result of the test is shown in Table 1. In this experiment, the error rate of defect length was $19.29 \%$, and the error rate of defect depth was $20.80 \%$.

Table 1. Comparison of measured value and actual value

\begin{tabular}{|c|c|c|c|c|c|}
\hline & No.1 & No.2 & No.3 & No.4 & Average value \\
\hline $\begin{array}{c}\text { Detection length } \\
(\mathrm{mm})\end{array}$ & 14.0 & 15.3 & 16.3 & 17.7 & - \\
\hline $\begin{array}{c}\text { Actual length } \\
\text { (mm) }\end{array}$ & 12.0 & 12.0 & 20.0 & 20.0 & $19.29 \%$ \\
\hline $\begin{array}{c}\text { Relative error rate } \\
\text { of length }\end{array}$ & $16.67 \%$ & $27.50 \%$ & $21.5 \%$ & $11.5 \%$ & - \\
\hline $\begin{array}{c}\text { Detection depth } \\
\text { (mm) }\end{array}$ & 3.6423 & 2.6762 & 2.7653 & 2.2269 & - \\
\hline $\begin{array}{c}\text { Actual depth } \\
\text { (mm) }\end{array}$ & 2.4000 & 2.4000 & 2.7000 & 2.7000 & $20.80 \%$ \\
\hline $\begin{array}{c}\text { Relative error rate } \\
\text { of depth }\end{array}$ & $51.76 \%$ & $11.51 \%$ & $2.42 \%$ & $17.52 \%$ & 2 \\
\hline
\end{tabular}




\section{Conclusions}

This paper discusses the weak magnetic detection of standard test blocks and verifies its effectiveness for standard test block detection. As the experimenter manually shifts the probe unevenly during the signal acquisition process of this experiment, it will bring errors, and the accuracy can be improved by automation. In the test, only the artificial holes were scanned, but the natural defects on the side interfered with the measurement, but the recognition and quantification of the artificial defects had little effect. Experiments show that the double probe has the smallest measurement error rate in the length direction, and the single probe has the smallest measurement error rate in the depth direction.

\section{Acknowledgements}

This work was financially supported by Chongqing Science and Technology Innovation Program for Social Undertakings and Livelihood Security (cstc2017shmsA00004).

\section{References}

[1] Wang H, Yu R, Zhang W, et al. Application Research On Weak Magnetic Testing On The Welds Of Small Diameter Tube[J]. Nondestructive Test, 2016.

[2] Xiao N, Yu R, Wang Y, et al. The Effect of Detection Direction on the Weak-Magnetism Inspection of Butt Weld[J]. Nondestructive Test, 2017.

[3] Xu Weijin. Study of micro magnetic detection technology for engine turbine disc[D]. Nanchang Hangkong University, 2014.

[4] Jin Yufei. Technical requirements and utilization of new reference test blocks for penetrant testing [J]. Nondestructive Test, 2003, 25(10):536-538.

[5] Tao Wangbin, Zhang Lipan, Song Kai.The mechanism study of the annulus reference block on magnetic particle testing[J]. Journal of Nanchang Institute of Aeronautical Technology(Natural Science),2005,19(3):60-64.

[6] Liang Jing,Han Bo.Research on the calibration method of ultrasonic responds of ultrasonic standard reference blocks[C].China Society of Mechanical Engineering Non-destructive Testing Branch Annual Meeting / International Non-destructive Testing Technology Exchange Seminar. 1999.

[7] Gao Songzhen, Liu Baicheng. Simulation and experiment of weak magnetic detection in stress concentration area of steel plate[J]. Nondestructive testing, 2011, 35(2):8-10.

[8] Guo Peng. Experiment research on nondestructive testing for wire ropes based on weak magnetic[D]. Yanshan University, 2012. [9] Zhaoming Z, Taihe S, Runqiao Y, et al. A New Approach to In-Service Coiled Tubing Improved Inspection Based Micro-Magnetic Technology[J]. Society of Petroleum Engineers , 2015, 17(3): 159-163.

[10] Shi R. Wire Rope Inspection Technology Research Based on Weak Magnetic[J]. Society of Petroleum Engineers , 2014, 35(1): 251-267.

[11] SHANIAVSKIY A, TOUSHENTSOV A L. Mechanisms of fatigue crack initiation and propagation in cast aluminum alloy AL5 of hydro pumps NP-89D in aircraft Tu-154M[J]. Engineering Failure Analysis, 2010, 17(3): 658 663.

[12] Yilai M, Li L. Research on internal and external defect identification of drill pipe based on weak magnetic inspection[J]. Insight-Non-Destructive Testing and Condition Monitoring, 2014, $56(1): 31-34$. 